\title{
Computation of transition matrices of positive linear electrical circuits
}

JEL: L62 DOI: 10.24136/atest.2019.147

Data zgłoszenia: 05.04.2019 Data akceptacji: 26.06.2019

A method is proposed for calculation of transition matrices of positive electrical circuits. It is shown that if the transition matrix is presented as finite series of the Metzler matrix with real distinct eigenvalues then the coefficients of the series are nonnegative function of time. The method is applied to positive linear electrical circuits.

Słowa kluczowe: positive, linear, electrical circuits, calculation, transition matrix.

\section{Introduction}

A dynamical system is called positive if its trajectory starting from any nonnegative initial state remains forever in the positive orthant for all nonnegative inputs. An overview of state of the art in positive theory is given in the monographs $[2,9]$. Variety of models having positive behavior can be found in engineering, especially in electrical circuits [17], economics, social sciences, biology and medicine, etc. [2, 9].

The positive electrical circuits have been analyzed in [4-8, 10 17]. The constructability and observability of standard and positive electrical circuits has been addressed in [5], the decoupling zeros in [6] and minimal-phase positive electrical circuits in [7]. A new class of normal positive linear electrical circuits has been introduced in [8] Positive fractional linear electrical circuits have been investigated in [11], positive linear systems with different fractional orders in [12, 13] and positive unstable electrical circuits in [14]. Zeroing of state variables in descriptor electrical circuits has been addressed in [16] and the realization problem of positive linear systems in [1].

In this paper a method for computation of transition matrices of positive linear electrical circuits will be presented and some specific properties of the coefficients of finite series presenting the transition matrices will be investigated.

The paper is organized as follows. In section 2 some basic definitions and theorems concerning matrices and Cayley-Hamilton theorem are recalled. The computation of the matrix function and the transition matrix are addressed in section 3 . The stability and computation of the transition matrices of positive linear electrical circuits are investigated in section 4 . Concluding remarks are given in section 5 .

The following notation will be used: $\mathfrak{R}$ - the set of real numbers, $\mathfrak{R}^{n \times m}$ - the set of $n \times m$ real matrices and $\mathfrak{R}^{n}=\mathfrak{R}^{n \times 1}$, $\mathfrak{R}_{+}^{n \times m}$ - the set of $n \times m$ matrices with nonnegative entries and $\mathfrak{R}_{+}^{n}=\mathfrak{R}_{+}^{n \times 1}, M_{n}$ - the set of $n \times n$ Metzler matrices (real matrices with nonnegative off-diagonal entries), $I_{n}$ - the $n \times n$ identity matrix, $A^{T}$ denotes the transpose of the matrix $\mathrm{A}$.

\section{Preliminaries}

The characteristic polynomial of the matrix $A \in \mathfrak{R}^{n \times n}$

$$
\varphi(\lambda)=\operatorname{det}\left[I_{n} \lambda-A\right]=\lambda^{n}+a_{n-1} \lambda^{n-1}+\ldots+a_{1} \lambda+a_{0}(2.1)
$$

and its minimal polynomial $\psi(\lambda)$ are related by $[2,15]$

$$
\psi(\lambda)=\frac{\varphi(\lambda)}{D(\lambda)}
$$

where $D(\lambda)$ is the greatest common divisor of entries of the adjoint matrix $\left[I_{n} \lambda-A\right]_{a d}$. If the eigenvalues $\lambda_{1}, \lambda_{2}, \ldots$, $\lambda_{n}$ of the matrix $A$ are distinct, i.e. $\lambda_{i} \neq \lambda_{j}$ if $i \neq j$, $i, j=1, \ldots, n$, then $D(\lambda)=1$ and $\Psi(\lambda)=\varphi(\lambda)[3,15]$

Consider a matrix $A \in \mathfrak{R}^{n \times n}$ with the minimal characteristic polynomial

$$
\Psi(\lambda)=\left(\lambda-\lambda_{1}\right)^{m_{1}}\left(\lambda-\lambda_{1}\right)^{m_{2}} \ldots\left(\lambda-\lambda_{1}\right)^{m_{r}},
$$

where $\lambda_{1}, \lambda_{2}, \ldots, \lambda_{r}$ are the eigenvalues of the matrix $A$ and $\sum_{i=1}^{r} m_{i}=m \leq n$.

It is assumed that the function $f(\lambda)$ is well-defined on spectrum $\sigma_{A}=\left\{\lambda_{1}, \lambda_{2}, \ldots \lambda_{r}\right\}$ of the matrix $A$, i.e.

$$
f\left(\lambda_{k}\right), f^{(1)}\left(\lambda_{k}\right)=\left.\frac{d f(\lambda)}{d \lambda}\right|_{\lambda=\lambda_{k}}, \ldots, f^{\left(m_{k}-1\right)}\left(\lambda_{k}\right)=\left.\frac{d f^{m_{k}-1}(\lambda)}{d \lambda^{m_{k}-1}}\right|_{\lambda=\lambda_{k}}, k=1, \ldots r
$$

are finite.

In this case the matrix $f(A)$ is well-defined and it is given by the Lagrange-Sylvester formula $[3,15]$

$$
f(A)=\sum_{i=1}^{r} Z_{i 1} f\left(\lambda_{i}\right)+Z_{i 2} f^{(1)}\left(\lambda_{i}\right)+\ldots Z_{i m_{i}} f^{\left(m_{i}-1\right)}\left(\lambda_{i}\right),
$$

where

$$
Z_{i j}=\sum_{k=j-1}^{m_{i}-1} \frac{\Psi_{i}(A)\left(A-\lambda_{i} I_{n}\right)^{k}}{(k-j+1) !(j-1) !} \frac{d^{k-j+1}}{d \lambda^{k-j+1}}\left[\frac{1}{\Psi_{i}(A)}\right]_{\lambda=\lambda_{i}}
$$

and

$$
\Psi_{i}(A)=\frac{\Psi(A)}{\left(\lambda-\lambda_{i}\right)^{m_{i}}}, \quad i=1, \ldots, r
$$


Theorem 2.1. Let

$$
\Psi(A)=\operatorname{det}\left[I_{n} \lambda-f(A)\right]=\lambda^{n}+a_{n-1} \lambda^{n-1}+\ldots+a_{1} \lambda+a_{0}
$$

be the minimal characteristic polynomial of the matrix $f(A)$. Then the matrix $f(A)$ satisfies its characteristic equation, i.e.

$$
[f(A)]^{n}+a_{n-1}[f(A)]^{n-1}+\ldots+a_{1}[f(A)]+a_{0} I_{n}=0 .
$$

Proof. Proof is given in [3, 15].

In particular case for $f(A)=A$ we obtain the classical Cayley-Hamilton theorem.

\section{Computation of the transition matrices}

Definition 3.1. The matrix $A=\left[a_{i j}\right] \in \mathfrak{R}^{n \times n}$ is call Metzler matrix if $a_{i j} \geq 0$ for $i \neq j$.

The set of $n \times n$ Metzler matrices will be denoted $M_{n}$. To simplify the notation it is assumed that $\lambda_{i} \neq \lambda_{j}$ for $i \neq j$.

Theorem 3.1. Let $A \in \mathfrak{R}^{n \times n}, f(A, t)$ be a matrix function welldefined on the spectrum $\sigma_{A}=\left\{\lambda_{1}, \lambda_{2}, \ldots \lambda_{r}\right\}$ of the matrix $A, t$ - time and it can be presented in the series form

$$
f(A, t)=\sum_{k=0}^{\infty} a_{k}(t) A^{k}
$$

where $a_{k}(t)$ are some coefficients.

Then

$$
f(A, t)=\sum_{k=0}^{n-1} c_{k}(t) A^{k}
$$

where

$$
\Delta_{0}(t)=\left|\begin{array}{cccc}
f\left(\lambda_{1} t\right) & \lambda_{1} & \ldots & \lambda_{1}{ }^{n-1} \\
f\left(\lambda_{2} t\right) & \lambda_{2} & \ldots & \lambda_{2}{ }^{n-1} \\
\vdots & \vdots & \ddots & \vdots \\
f\left(\lambda_{n} t\right) & \lambda_{n} & \ldots & \lambda_{n}{ }^{n-1}
\end{array}\right|, \Delta_{1}(t)=\left|\begin{array}{cccc}
1 & f\left(\lambda_{1} t\right) & \ldots & \lambda_{1}{ }^{n-1} \\
1 & f\left(\lambda_{2} t\right) & \ldots & \lambda_{2}{ }^{n-1} \\
\vdots & \vdots & \ddots & \vdots \\
1 & f\left(\lambda_{n} t\right) & \ldots & \lambda_{n}{ }^{n-1}
\end{array}\right|, \ldots
$$

$$
\Delta_{n-1}(t)=\left|\begin{array}{cccc}
1 & \lambda_{1} & \ldots & \lambda_{1}{ }^{n-2} f\left(\lambda_{1} t\right) \\
1 & \lambda_{2} & \ldots & \lambda_{2}{ }^{n-2} \\
\vdots & \vdots & \ddots & \vdots \\
1 & \lambda_{n} & \ldots & \lambda_{n}{ }^{n-2} f\left(\lambda_{n} t\right)
\end{array}\right|, \quad \Delta=\left|\begin{array}{cccc}
1 & \lambda_{1} & \ldots & \lambda_{1}{ }^{n-1} \\
1 & \lambda_{2} & \ldots & \lambda_{2}{ }^{n-1} \\
\vdots & \vdots & \ddots & \vdots \\
1 & \lambda_{n} & \ldots & \lambda_{n}{ }^{n-1}
\end{array}\right| .
$$

Proof. Using the classical Cayley-Hamilton theorem we can eliminate in (3.1) the matrix $A^{k}$ for $k=n, n+1, \ldots$ and obtain (3.2). It is well-known $[3,15]$ that $(3.2)$ is also satisfied on the spectrum of the matrix $A$

$$
f\left(\lambda_{k}, t\right)=\sum_{j=0}^{n-1} c_{j}(t) \lambda^{k} \quad \text { for } k=1, \ldots, n .
$$

The relations (3.4) can be written in the form

$$
\left[\begin{array}{cccc}
1 & \lambda_{1} & \ldots & \lambda_{1}{ }^{n-1} \\
1 & \lambda_{2} & \ldots & \lambda_{2}{ }^{n-1} \\
\vdots & \vdots & \ddots & \vdots \\
1 & \lambda_{n} & \ldots & \lambda_{n}{ }^{n-1}
\end{array}\right]\left[\begin{array}{c}
c_{0}(t) \\
c_{1}(t) \\
\vdots \\
\left.c_{n-1} t\right)
\end{array}\right]=\left[\begin{array}{c}
f\left(\lambda_{1} t\right) \\
f\left(\lambda_{2} t\right) \\
\\
f\left(\lambda_{n} t\right)
\end{array}\right]
$$
(3.3).

Using the well-known the Cramer formula to (3.5) we obtain

The matrix $e^{A t}$ is called transition matrix of the linear systems.

Theorem 3.2. If $A \in M_{n}$ has distinct eigenvalues $\lambda_{1}, \lambda_{2}, \ldots$, $\lambda_{n}$ than the coefficients $c_{k}(t), k=0,1, \ldots n-1$ in the formula

$$
e^{A t}=\sum_{k=0}^{n-1} c_{k}(t) A^{k}
$$

satisfy the following conditions:

$$
\begin{aligned}
c_{k}(0) & =\left\{\begin{array}{l}
1 \text { for } k=0 \\
0 \text { for } k=1, \ldots, n-1
\end{array}\right. \\
\left.\frac{d c_{k}(t)}{d t}\right|_{t=0} & = \begin{cases}1 & \text { for } k=0 \\
0 & \text { for } k=0,2, \ldots, n-1\end{cases}
\end{aligned}
$$

and

$$
c_{k}(t) \geq 0 \text { for } t \geq 0 \text { and } k=0,1, \ldots, n-1 \text {. }
$$

Proof. The equality (3.6) follows from (3.2) for and are defined by (3.3). From (3.3) we have

$$
\begin{gathered}
\left.c_{0}(t)\right|_{t=0}=\frac{\Delta_{0}(0)}{\Delta}=1 \text { and }\left.c_{k}(t)\right|_{t=0}=\frac{\Delta_{k}(0)}{\Delta}=\frac{0}{\Delta}=0 \\
\text { for } k=0,1, \ldots n-1
\end{gathered}
$$

since for $\quad f(A, t)=e^{A t},\left.e^{\lambda_{k} t}\right|_{t=0}=1, k=1, \ldots, n$, $\Delta_{0}(0)=\Delta$ and $\Delta_{k}(0)$ for $k=1, \ldots, n-1$ has two identical columns.

Note that $\left.\frac{d}{d t} e^{\lambda_{k^{t}}}\right|_{t=0}=\lambda_{k}$ for $k=1, \ldots, n$, and

$\left.\frac{d c_{k}(t)}{d t}\right|_{t=0} \frac{\left.\frac{d \Delta(t)}{d t}\right|_{t=0}}{\Delta}= \begin{cases}\frac{\Delta_{k}(0)}{\Delta}=\frac{\Delta}{\Delta}=1 & \text { for } k=1 \\ \frac{\Delta_{k}(0)}{\Delta}=\frac{\Delta}{\Delta}=0 & \text { for } k=0,2, \ldots, n-1\end{cases}$ 
since $\Delta_{k}(0)$ for $k=0,2, \ldots, n-1$ has two identical columns.

The condition (3.9) is satisfied since

$$
\begin{gathered}
c_{k}\left(t_{1}\right) c_{k}\left(t_{2}\right)=\frac{c_{k}\left(t_{1}\right) c_{k}\left(t_{2}\right)}{\Delta^{2}} \geq 0 \quad \text { for } k=0,1, \ldots, n-1 \\
\text { and } t_{1}, t_{2} \in[0, \infty] .
\end{gathered}
$$

Example 3.1. Consider the matrix $e^{A t}$ for

$$
\left[\begin{array}{cc}
-1 & 0 \\
1 & -2
\end{array}\right] \in M_{2}
$$

with the eigenvalues $\lambda_{1}=-1, \lambda_{2}=-2$.

By Theorem 3.2 we have

$$
e^{A t}=c_{0}(t) I_{2}+c_{1}(t) A
$$

It will be shown that

$c_{0}(t)>0$ for $t \geq 0, c_{0}(0)=1, \lim _{t \rightarrow \infty} c_{0}(t)=0$

and

$c_{1}(t) \geq 0$ for $t \geq 0, c_{1}(0)=1, \lim _{t \rightarrow \infty} c_{1}(t)=0$.

Using (3.3) for (3.13) we obtain

$$
c_{0}(t)=\frac{\left|\begin{array}{ll}
e^{\lambda_{1} t} & \lambda_{1} \\
e^{\lambda_{2} t} & \lambda_{2}
\end{array}\right|}{\left|\begin{array}{ll}
1 & \lambda_{1} \\
1 & \lambda_{2}
\end{array}\right|}=\frac{1}{\lambda_{2}-\lambda_{1}}\left(\lambda_{2} e^{\lambda_{1} t}-\lambda_{1} e^{\lambda_{2} t}\right)=2 e^{-t}-e^{-2 t}>0
$$$$
\text { for } t \geq 0
$$

and

$$
c_{1}(t)=\frac{\left|\begin{array}{ll}
1 & e^{\lambda_{1} t} \\
1 & e^{\lambda_{2} t}
\end{array}\right|}{\left|\begin{array}{ll}
1 & \lambda_{1} \\
1 & \lambda_{2}
\end{array}\right|}=\frac{1}{\lambda_{2}-\lambda_{1}}\left(e^{\lambda_{2} t}-e^{\lambda_{1} t}\right)=e^{-t}-e^{-2 t} \geq 0
$$

Note that

$$
\begin{gathered}
\frac{d c_{0}(t)}{d t}=\frac{\lambda_{1} \lambda_{2}}{\lambda_{2}-\lambda_{1}}\left(e^{\lambda_{1} t}-e^{\lambda_{2} t}\right)=2 e^{-2 t}-2 e^{-t}>0 \text { for } \\
t \geq 0
\end{gathered}
$$

and

$$
\begin{gathered}
\frac{d c_{1}(t)}{d t}=\frac{1}{\lambda_{2}-\lambda_{1}}\left(\lambda_{2} e^{\lambda_{2} t}-\lambda_{1} e^{\lambda_{1} t}\right)=2 e^{-2 t}-e^{-t}>0 \text { for } \\
t \geq 0
\end{gathered}
$$

$$
\begin{gathered}
\frac{d c_{1}(t)}{d t} \geq 0 \text { for } 0 \leq t \leq \frac{1}{\lambda_{1}-\lambda_{2}} \ln \frac{\lambda_{2}}{\lambda_{1}} \text { and } \frac{d c_{1}(t)}{d t}<0 \\
\text { for } 0 \leq t \leq \frac{1}{\lambda_{1}-\lambda_{2}} \ln \frac{\lambda_{2}}{\lambda_{1}} .
\end{gathered}
$$

Therefore, the conditions of Theorem 3.2 are satisfied (see Fig. 3.1).
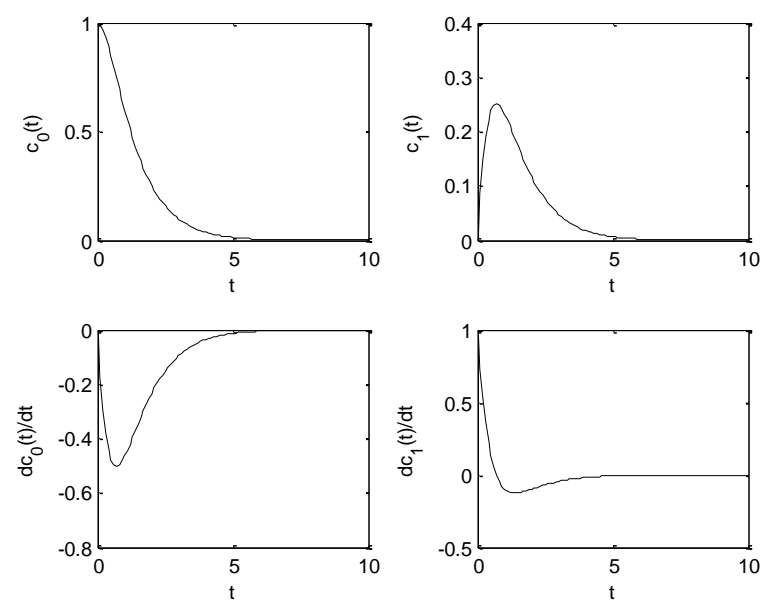

Fig. 3.1. Plots of coefficients $c_{k}(t)$ and their derivatives

Example 3.2. Consider the matrix function $e^{A t}$ for $A \in M_{3}$ with the eigenvalues $\lambda_{1}=-1, \lambda_{2}=-2, \lambda_{3}=-3$.

Note that the matrix $A \in M_{3}$ is given by the set of matrices

$$
A=P\left[\begin{array}{ccc}
-1 & 0 & 0 \\
0 & -2 & 0 \\
0 & 0 & -3
\end{array}\right] P^{-1}
$$

for $P \in \mathfrak{R}^{3 \times 3}$ monomial matrix.

Using(3.3) for (3.10) we obtain

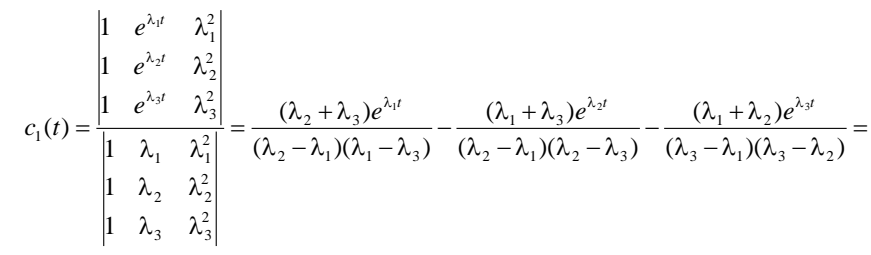
$=\frac{5}{2} e^{-t}-4 e^{-2 t}+\frac{3}{2} e^{-3 t}$

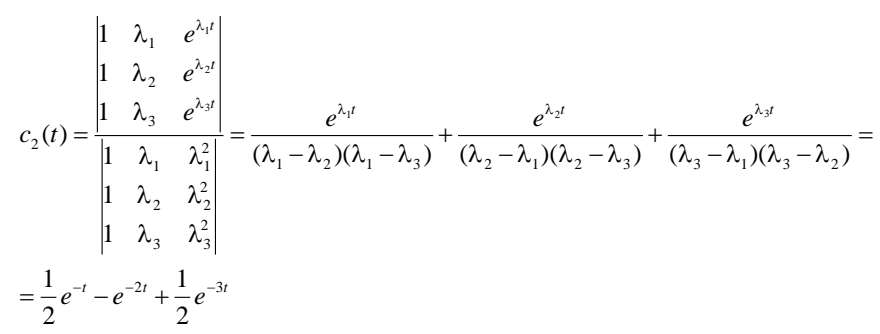

from (3.18) it follows that 
From (3.21) - (3.23) is follows that $c_{0}(0)=1, c_{1}(0)=0$, $c_{2}(0)=0$ and $c_{k}(t) \geq 0$ for $t \geq 0$.

Therefore the conditions of Theorem 3.2 are satisfied (see Fig. 3.2).
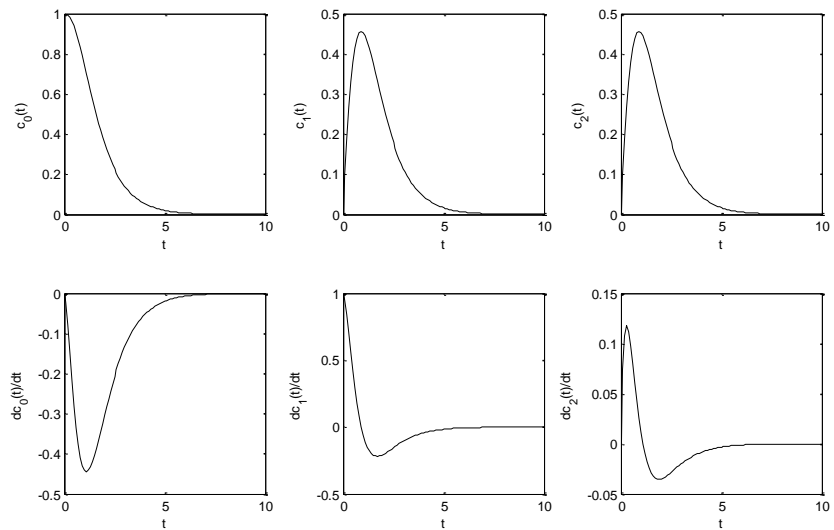

Fig. 3.2. Plots of coefficients $c_{k}(t)$ and their derivatives

4.The stability of positive electrical circuits and computation of their transition matrices

Consider the linear continuous-time electrical circuit described by the state equations

$$
\begin{aligned}
& \dot{x}(t)=A x(t)+B u(t), \\
& y(t)=C x(t)+D u(t),
\end{aligned}
$$

where $x(t) \in \mathfrak{R}^{n}, u(t) \in \mathfrak{R}^{m}, y(t) \in \mathfrak{R}^{p}$ are the state, input and output vectors and $A \in \mathfrak{R}^{n \times n}, B \in \mathfrak{R}^{n \times m}, C \in \mathfrak{R}^{p \times n}$, $D \in \mathfrak{R}^{p \times m}$.

Definition 4.1. $[2,10,17]$ The electrical circuit $(4.1)$ is called (internally) positive if $\quad x(t) \in \mathfrak{R}_{+}^{n} \quad$ and $\quad y=y(t) \in \mathfrak{R}_{+}^{p}$, $t \in[0,+\infty]$ for any $x_{0}=x(0) \in \mathfrak{R}_{+}^{n}$ and every $u(t) \in \mathfrak{R}_{+}^{m}$, $t \in[0,+\infty]$.

Theorem 4.1. $[2,10,17]$ The electrical circuit (2.1) is positive if and only if

$$
A \in M_{n}, B \in \mathfrak{R}_{+}^{n \times m}, C \in \mathfrak{R}_{+}^{p \times n}, D \in \mathfrak{R}_{+}^{p \times m} .
$$

It is well-known $[2,10,17]$ that any linear electrical circuit composed of resistors, coils, capacitors and voltage (current) sources can be described by the state equations (4.1). Usually as the state variables $x_{1}(t), \ldots, x_{n}(t)$ (the components of the state vector $x(t))$ the currents in the coils and voltages on the capacitors are chosen.

Theorem 4.2. The linear electrical circuit composed of resistors, coils and voltage sources is positive for any values of the resistances, inductances and source voltages if the number of coils is less or equal to the number of its linearly independent meshes and the direction of the mesh currents are consistent with the directions of the mesh source voltages.

Proof. Proof is given in [17].

Theorem 4.3. The $R, L, C$, e electrical circuits are not positive for any values of their resistances, inductances, capacitances and source voltages if at least one their branch contains coil and capacitor.
Proof. Proof is given in [17].

Definition 4.2. [17] The positive electrical circuit (4.1) is called asymptotically stable if

$$
\lim _{t \rightarrow \infty} x(t)=0 \text { for all } x_{0} \in \mathfrak{R}_{+}^{n} \text {. }
$$

Theorem 4.4 [17] The positive electrical circuit (4.1) is asymptotically stable if and only if

$$
\operatorname{Re} \lambda_{k}<0 \text { for } k=1, \ldots, n
$$

where $\lambda_{k}$ is the eigenvalue of the matrix $A \in M_{n}$ and

$$
\operatorname{det}\left[I_{n} \lambda-A\right]=\left(\lambda-\lambda_{1}\right)\left(\lambda-\lambda_{2}\right) \ldots\left(\lambda-\lambda_{n}\right)
$$

Theorem 4.5. $[3,15]$ A symmetric matrix $A \in \mathfrak{R}^{n \times n}\left(A^{T}=A\right)$ has only real eigenvalues $\lambda_{k}, k=1, \ldots, n$.

Theorem 4.6. Let $\bar{\lambda}_{k}, k=1, \ldots, n$ be real eigenvalues of the symmetric Hurwitz matrix $\bar{A} \in \mathfrak{R}^{n \times n}$. Then the matrix

$$
\begin{aligned}
A=D \bar{A} \in \mathfrak{R}^{n \times n}, & D=\operatorname{diag}\left[\begin{array}{lll}
d_{1} & \cdots & d_{n}
\end{array}\right], d_{k}>0, \\
k=1, \ldots, n &
\end{aligned}
$$

has also only real eigenvalues

$$
\lambda_{k}=d_{k} \bar{\lambda}_{k} \text { for } k=1, \ldots, n \text {. }
$$

Proof. By Theorem 4.5 the symmetric matrix $\bar{A}$ has only real eigenvalues $\bar{\lambda}_{k}, k=1, \ldots, n$. Note that

$$
\operatorname{det}\left[I_{n} \lambda-D \bar{A}\right]=\operatorname{det}\left\{D\left[D^{-1} \lambda-\bar{A}\right]\right\}=\operatorname{det} D \operatorname{det}\left[D^{-1} \lambda-\bar{A}\right]
$$

and

$$
\begin{aligned}
& \operatorname{det}\left[I_{n} \lambda-D \bar{A}\right]=0 \text { if and only if } \\
& \operatorname{det}\left[D^{-1} \lambda-\bar{A}\right]=\operatorname{det}\left[I_{n} \bar{\lambda}-\bar{A}\right]=0
\end{aligned}
$$

The equality (4.9) implies (4.7). $\square$

Example 4.1. Consider the electrical circuit shown on Figure 4.1 with given resistances $R_{1}, R_{2}, R_{3}$, inductances $L_{1}, L_{2}$ and source voltages $e_{1}, e_{2}$.

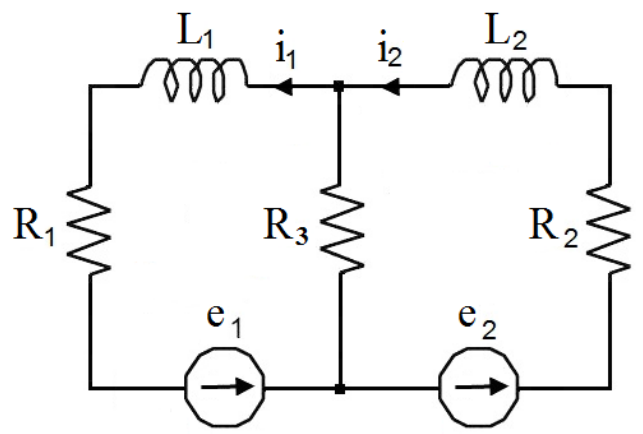

Fig. 4.1. Electrical circuit with inductances

Using the Kirchhoff's laws we may write the equations 


$$
\begin{aligned}
& e_{1}=R_{1} i_{1}+L_{1} \frac{d i_{1}}{d t}+R_{3}\left(i_{1}-i_{2}\right) \\
& e_{2}=R_{2} i_{2}+L_{2} \frac{d i_{2}}{d t}+R_{3}\left(i_{2}-i_{1}\right)
\end{aligned}
$$

which can be written in the form

$$
\frac{d}{d t}\left[\begin{array}{l}
i_{1} \\
i_{2}
\end{array}\right]=A_{1}\left[\begin{array}{l}
i_{1} \\
i_{2}
\end{array}\right]+B_{1}\left[\begin{array}{l}
e_{1} \\
e_{2}
\end{array}\right],
$$

where

$$
A_{1}=\left[\begin{array}{cc}
-\frac{R_{1}+R_{3}}{L_{1}} & \frac{R_{3}}{L_{1}} \\
\frac{R_{3}}{L_{2}} & -\frac{R_{2}+R_{3}}{L_{2}}
\end{array}\right], B_{1}=\left[\begin{array}{cc}
\frac{1}{L_{1}} & 0 \\
0 & \frac{1}{L_{2}}
\end{array}\right]
$$

The electrical circuit is positive since the matrix $A_{1}$ is a Metzler matrix and the matrix $B_{1}$ has nonnegative entries.

Using (4.12) for $R_{1}=R_{2}=2, R_{3}=1, L_{1}=L_{2}=2$ we obtain

$$
A_{1}=\left[\begin{array}{cc}
-\frac{R_{1}+R_{3}}{L_{1}} & \frac{R_{3}}{L_{1}} \\
\frac{R_{3}}{L_{2}} & -\frac{R_{2}+R_{3}}{L_{2}}
\end{array}\right]=\left[\begin{array}{cc}
-\frac{3}{2} & \frac{1}{2} \\
\frac{1}{2} & -\frac{3}{2}
\end{array}\right]
$$

and

$\operatorname{det}\left[I_{2} \lambda-A_{1}\right]=-\left|\begin{array}{cc}\lambda+\frac{3}{2} & -\frac{1}{2} \\ -\frac{1}{2} & \lambda+\frac{3}{2}\end{array}\right|=\lambda^{2}+3 \lambda+2=(\lambda+1)(\lambda+2)$.

The eigenvalues of the matrix (4.13) are: $\lambda_{1}=-1, \lambda_{2}=-2$.

Using the results of Example 3.1 we obtain

$$
\begin{aligned}
& e^{A_{1} t}=c_{0}(t) I_{2}+c_{1}(t) A_{1}=\left(2 e^{-t}-e^{-2 t}\right)\left[\begin{array}{ll}
1 & 0 \\
0 & 1
\end{array}\right]+e^{-t}-e^{-2 t}\left[\begin{array}{cc}
-3 / 2 & 1 / 2 \\
1 / 2 & -3 / 2
\end{array}\right]= \\
& =\left[\begin{array}{ll}
\frac{e^{-2 t}+e^{-t}}{2} & \frac{-e^{-2 t}+e^{-t}}{2} \\
\frac{-e^{-2 t}+e^{-t}}{2} & \frac{e^{-2 t}+e^{-t}}{2}
\end{array}\right]
\end{aligned}
$$

Example 4.2. Consider the electrical circuit shown on Figure 4.2 with given resistances $R_{k}, k=1, \ldots, 4$, inductances $L_{1}, L_{2}$, capacitance $C$ and source voltages $e_{j}, j=1,2,3$.

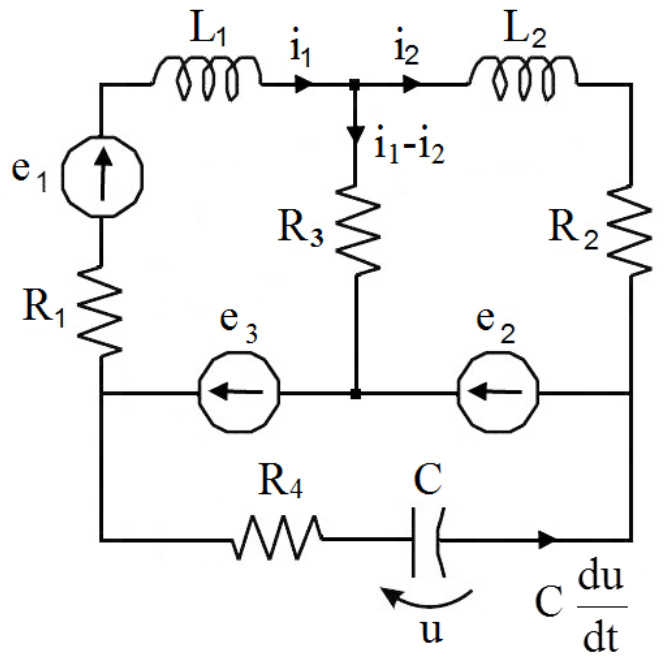

Fig. 4.2. Electrical circuit of Example 4.2

Using the Kirchhoff's laws we can write the equations

$$
\begin{gathered}
e_{1}+e_{3}-\left(R_{1}+R_{3}\right) i_{1}+R_{3} i_{2}=L_{1} \frac{d i_{1}}{d t}, \\
e_{2}-\left(R_{2}+R_{3}\right) i_{2}+R_{3} i_{1}=L_{2} \frac{d i_{1}}{d t}, \\
e_{2}+e_{3}-u=R_{4} C \frac{d u}{d t}
\end{gathered}
$$

which can be written in the form

$$
\frac{d}{d t}\left[\begin{array}{l}
i_{1} \\
i_{2} \\
u
\end{array}\right]=A_{2}\left[\begin{array}{l}
i_{1} \\
i_{2} \\
u
\end{array}\right]+B_{2}\left[\begin{array}{l}
e_{1} \\
e_{2} \\
e_{3}
\end{array}\right]
$$

where

$$
\begin{gathered}
A_{2}=\left[\begin{array}{ccc}
-\frac{R_{1}+R_{3}}{L_{1}} & \frac{R_{3}}{L_{1}} & 0 \\
\frac{R_{3}}{L_{2}} & -\frac{R_{2}+R_{3}}{L_{2}} & 0 \\
0 & 0 & -\frac{1}{R_{4} C}
\end{array}\right] \\
B_{2}=\left[\begin{array}{ccc}
\frac{1}{L_{1}} & 0 & \frac{1}{L_{1}} \\
0 & \frac{1}{L_{2}} & 0 \\
0 & \frac{1}{R_{4} C} & \frac{1}{R_{4} C}
\end{array}\right] .
\end{gathered}
$$

The electrical circuit is positive since $A_{2} \in M_{3}$ and $B_{2} \in \mathfrak{R}_{+}^{3 \times 3}$ for all values of the resistances, inductances and capacitance.

Using (4.18) for $R_{1}=R_{2}=2, R_{3}=R_{4}=1, L_{1}=L_{2}=1$, $C_{1}=1$ we obtain 
$A_{2}=\left[\begin{array}{ccc}-\frac{R_{1}+R_{3}}{L_{1}} & \frac{R_{3}}{L_{1}} & 0 \\ \frac{R_{3}}{L_{2}} & -\frac{R_{2}+R_{3}}{L_{2}} & 0 \\ 0 & 0 & -\frac{1}{R_{4} C}\end{array}\right]=\left[\begin{array}{ccc}-3 & 1 & 0 \\ 1 & -3 & 0 \\ 0 & 0 & -1\end{array}\right]$

and

$\operatorname{det}\left[I_{3} \bar{\lambda}-A_{2}\right]=\left|\begin{array}{ccc}\bar{\lambda}+3 & -1 & 0 \\ -1 & \bar{\lambda}+2 & 0 \\ 0 & 0 & \bar{\lambda}+1\end{array}\right|=(\bar{\lambda}+1)\left(\bar{\lambda}^{2}+6 \bar{\lambda}+8\right)=(\bar{\lambda}+1)(\bar{\lambda}+2)(\bar{\lambda}+4)$.

The eigenvalues of the matrix (4.19) are: $\bar{\lambda}_{1}=-1, \bar{\lambda}_{2}=-2$, $\bar{\lambda}_{3}=-4$.

Using the results of Example 3.2 we obtain

$$
\begin{aligned}
e^{A_{2} t}= & \bar{c}_{0}(t) I_{3}+\bar{c}_{1}(t) A_{2}+\bar{c}_{2}(t) A_{2}^{2}=\left(3 e^{-t}-3 e^{-2 t}+e^{-3 t}\right)\left[\begin{array}{lll}
1 & 0 & 0 \\
0 & 1 & 0 \\
0 & 0 & 1
\end{array}\right]+ \\
& +\left(\frac{5}{2} e^{-t}-4 e^{-2 t}+\frac{3}{2} e^{-3 t}\right)\left[\begin{array}{ccc}
-3 & 1 & 0 \\
1 & -3 & 0 \\
0 & 0 & -1
\end{array}\right]+ \\
& +\left(\frac{1}{2} e^{-t}-e^{-2 t}+\frac{1}{2} e^{-3 t}\right)\left[\begin{array}{ccc}
-3 & 1 & 0 \\
1 & -3 & 0 \\
0 & 0 & -1
\end{array}\right]= \\
= & {\left[\begin{array}{ccc}
\frac{41}{2} e^{-t}-31 e^{-2 t}+\frac{23}{2} e^{-3 t} & -\frac{25}{2} e^{-t}-20 e^{-2 t}+\frac{15}{2} e^{-3 t} & 0 \\
-\frac{25}{2} e^{-t}-20 e^{-2 t}+\frac{15}{2} e^{-3 t} & \frac{41}{2} e^{-t}-31 e^{-2 t}+\frac{23}{2} e^{-3 t} & 0 \\
0 & 0 & 3 e^{-t}-3 e^{-2 t}+e^{-3 t}
\end{array}\right] }
\end{aligned}
$$

\section{Concluding remarks}

It has been shown that every matrix function $f(A, t)$ welldefined on its spectrum can be presented by (3.2) and the coefficients are given by (3.3) (Theorem 3.1). If the matrix $A \in M_{n}$ has distinct eigenvalues then the coefficients in the formula (3.6) satisfy the conditions (3.7) - (3.9) (Theorem 3.2). The formula (3.6) allows to compute the transition matrix $e^{A t}$ for a given matrix $A \in M_{n}$ with distinct eigenvalues. The efficiency of the method has been demonstrated on examles of positive electrical circuits. The considerations can be extended to matrices with multiple real and complex eigenvalues. An extension of these considerations is also possible for positive fractional linear electrical circuits.

\section{References}

1. L. Benvenuti, L. Farina, "A tutorial on the positive realization problem," IEEE Trans. on Automatic Control, vol. 49, no. 5, pp. 651-664, 2004.

2. L. Farina, S. Rinaldi, Positive Linear Systems; Theory and Applications, J. Wiley, New York, 2000.
3. F.R. Gantmacher, The Theory of Matrices. Chelsea Pub. Comp., London, 1959.

4. T. Kaczorek, "A class of positive and stable time-varying electrical circuits," Electrical Review, vol. 91, no. 5, pp. 121-124, 2015.

5. T. Kaczorek, "Constructability and observability of standard and positive electrical circuits," Electrical Review, vol. 89, no. 7, pp. 132-136, 2013.

6. T. Kaczorek, "Decoupling zeros of positive continuous-time linear systems and electrical circuits," Advances in Systems Science, vol. 240, pp. 1-15, 2014.

7. T. Kaczorek, "Minimal-phase positive electrical circuits," Electrical Review, vol. 92, no. 3, pp. 182-189, 2016.

8. T. Kaczorek, "Normal positive electrical circuits," IET Circuits Theory and Applications, vol. 9, no. 5, pp. 691-699, 2015.

9. T. Kaczorek, Positive $1 D$ and $2 D$ Systems, Springer-Verlag, London, 200

10. T. Kaczorek, "Positive electrical circuits and their reachability," Archives of Electrical Engineering, vol. 60, no. 3, pp. 283-301, 2011.

11. T. Kaczorek, "Positive fractional linear electrical circuits," Proceedings of SPIE, vol. 8903, Bellingham WA, USA, Art. No 3903-35.

12. T. Kaczorek, "Positive linear systems with different fractional orders.," Bull. Pol. Acad. Sci. Techn., vol. 58, no. 3, pp. 453458, 2010.

13. T. Kaczorek, "Positive systems consisting of $n$ subsystems with different fractional orders," IEEE Trans. Circuits and Systems regular paper, vol. 58, no. 6, pp. 1203-1210, June 2011.

14. T. Kaczorek, "Positive unstable electrical circuits," Electrical Review, vol. 88, no. 5a, pp. 187-192, 2012.

15. T. Kaczorek, Vectors and Matrices in Automation and Electrotechnics, WNT, Warsaw, 1998 (in Polish).

16. T. Kaczorek, "Zeroing of state variables in descriptor electrical circuits by state-feedbacks," Electrical Review, vol. 89, no. 10, pp. 200-203, 2013.

17. T. Kaczorek, K. Rogowski, Fractional Linear Systems and Electrical Circuits. Studies in Systems, Decision and Control, vol. 13, Springer, 2015.

„The studies have been carried out in the framework of work No. S/WE/1/2016 and financed from the funds for science by the Polish Ministry of Science and Higher Education"

\section{Obliczanie macierzy tranzycji \\ dodatnich liniowych obwodów elektrycznych}

Podano metodę obliczania macierzy tranzycji dodatnich obwodów elektrycznych. Wykazano, że jeżeli macierz tranzycji ma postać skończonego szeregu macierzy Metzlera o różnych rzeczywistych wartościach własnych to współczynniki tego szeregu są nieujemnymi funkcjami czasu. Metoda ta została zastosowana do analizy dodatnich obwodów elektrycznych.

Słowa kluczowe: dodatni, liniowy, obwód elektryczny, obliczanie ,macierz tranzycji.

Author:

Tadeusz KACZOREK - Bialystok University of Technology, Faculty of Electrical Engineering Wiejska 45D, 15-351 Bialystok, e-mail: kaczorek@isep.pw.edu.pl 